Pneumologe $2015 \cdot 12: 461-463$

DOI 10.1007/s10405-015-0007-0

Online publiziert: 23. September 2015

(c) Springer-Verlag Berlin Heidelberg 2015

CrossMark

\section{T. Nüßlein}

Klinik für Kinder- und Jugendmedizin, Gemeinschaftsklinikum Mittelrhein gGmbH, Koblenz, Deutschland
Fortschritt wird begünstigt durch Unzufriedenheit mit der Ausgangssituation. Deshalb ist die Pädiatrie mit all ihren besonderen Anforderungen hier und da besonders motiviert, neue Methoden einzuführen. Dieses Heft ist Techniken in der Pneumologie gewidmet, die an der Schwelle zur weiten Verbreitung im klinischen Einsatz stehen.

Schnell lassen sich Aspekte aufzählen, die den pädiatrischen Pneumologen in besonderer Weise fordern. Ein definiertes Atemmanöver, das man dem erwachsenen Patienten mit einem Satz erläutert, ist für das Kindergartenkind möglicherweise gar nicht umsetzbar. Untersuchungen, die mit langer Dauer, großem Lärm oder Schmerzen einhergehen, erfordern bei heranwachsenden Patienten oft eine Sedierung oder gar Narkose. Sogar physiologische Parameter, etwa die hohe Atemfrequenz kleiner Kinder, begrenzen den Einsatz von Methoden, die in der Medizin für Erwachsene gang und gäbe sind.

Entsprechend groß ist in der pädiatrischen Pneumologie die Motivation, solche Hürden zu überwinden. Die Wünsche lassen sich recht leicht aus den Begrenzungen ableiten:

- Im Idealfall lassen sich die Techniken auch ohne Kooperation des Patienten durchführen.

- Bei progredienten Krankheitsverläufen ist die frühzeitige Einordnung der Problematik anzustreben, um Folgeschäden gar nicht aufkommen zu lassen.

- Wann immer möglich, sollten Untersuchungen vermieden werden, die eine Sedierung oder Narkose erfordern.

- Die Abwägung von Nutzen und Nebenwirkungen ist für junge Patienten noch kritischer durchzuführen als für Erwachsene, beispielsweise im Hinblick auf Strahlenexposition.

Die Beiträge dieses Sonderheftes stellen Methoden vor, die die Probephase hinter sich haben, punktuell bereits im klinischen Routinebetrieb zur Anwendung kommen, zumeist an der Schwelle zur allgemeinen Anwendung stehen. Trotz der besonderen Motivation der pädiatrischen Pneumologie beschränkt sich die Anwendung schon jetzt natürlich keinesfalls auf das Kindes- und Jugendalter.

Kinder im Vorschulalter haben auch ohne Grunderkrankung bis zu zehn Infekte pro Jahr. Entsprechend häufig sind Diagnosen mit einem bakteriellen oder viralen Erreger als Ursache. Folke Brinkmann und Tobias Ankermann bewerten in ihrem Beitrag den Einsatz PCR-basierter Erregerdiagnostik für Atemwegserkrankungen bei Kindern.

Die Atopie manifestiert sich beim allergrößten Teil betroffener Patienten in den ersten Lebensjahren, die meisten Erwachsenen mit einem Asthma bronchiale haben eine viele Jahre zurückreichende Krankengeschichte. Die Leitlinie fordert - und die tägliche Arbeit untermauert diesen Anspruch -, dass in der Phase der Diagnosestellung auch nach Allergien gefahndet wird. Hier bietet die Bestimmung von IgE für rekombinante Allergene neue Perspektiven. Lars Lange stellt den Stand der Dinge in seinem Beitrag dar.

Für die Darstellung auch kleinster Strukturen in der Lunge, im Einzelfall auch dreidimensional rekonstruiert, erfüllt die Computertomografie des Thorax nahezu alle Wünsche des Pneumologen. Jedoch hat die Strahlenexposition, die mit zunehmendem Alter immer weni- ger relevant ist, für das Kind mit noch einigen Jahrzehnten Lebenserwartung eine unvergleichlich höhere Relevanz. Christoph Heyer stellt mit seinem Beitrag zur Kernspintomografie der Lunge eine Option vor, die schon jetzt beispielsweise für die Diagnose Mukoviszidose regelmäßig angewandt wird.

Die großen Schritte in der Entwicklung der Lungenfunktionsdiagnostik sind schnell aufgezählt. Limitierender Faktor bei den weit verbreiteten Techniken ist das Erfordernis der Kooperation des Patienten. Darüber hinaus mangelt es an Methoden, die über das gesamte Leben vom Kleinkindalter bis ins Greisenalter anwendbar sind. Abhilfe zeichnet sich mit der Weiterentwicklung einer alten Rückatmungsmethode ab, der Bestimmung des Lung Clearance Index mittels Multiple Breath Washout. Kim Husemann und Martin Kohlhäufl stellen alles Wissenswerte dazu in ihrem Beitrag dar.

Auch therapeutisch ist Fortschritt notwendig und auch erkennbar. Die Entfernung von Fremdkörpern mittels Bronchoskop basiert traditionell auf einer starren Technik mit Narkose. Der inzwischen weitestgehende Verzicht auf diese Untersuchungstechnik mit diagnostischer Fragestellung führt zur drastischen Abnahme der Frequenz solcher Untersuchungen und damit auch der breiten Verfügbarkeit. Der Ansporn in den letzten Jahren war entsprechend groß, Fremdkörper auch mit flexibler Technik aus den Atemwegen zu entfernen. Dirk Schramm stellt diese Perspektive in seinem Beitrag „Fremdkörperaspiration im Kindesalter" vor.

Damit leuchten die State-of-the-ArtArtikel dieses Sonderheftes die wesentlichen Bereiche der Pneumologie aus, 


\section{Einführung zum Thema}

zwar überwiegend aus der pädiatrischen Perspektive, deswegen möglicherweise besonders interessant für die Pneumologen in der Leserschaft, die überwiegend erwachsene Patienten betreuen.

Eine angenehme und nutzbringende Lektüre wünscht Ihnen

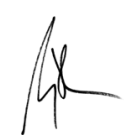

Thomas Nüßlein

\section{Korrespondenzadresse}

\section{PD Dr. T. Nüßlein}

Klinik für Kinder- und Jugendmedizin, Gemeinschaftsklinikum Mittelrhein gGmbH Koblenzer Straße 115-155, 56073 Koblenz,

Deutschland

thomas.nuesslein@gk.de

Interessenkonflikt. T. Nüßlein gibt an, dass kein

Interessenkonflikt besteht.

\section{Hier steht eine Anzeige}

\section{黛 Springer}


Hier steht eine Anzeige.

刑 Springer 